\title{
Visible and infrared photoluminescence from erbium-doped silicon nanocrystals produced by rf sputtering
}

\author{
M.F. Cerqueira ${ }^{1}$, M. Losurdo ${ }^{2}$, T. Monteiro ${ }^{3}$, M. Stepikova ${ }^{4}$, M. J. Soares ${ }^{3}$, M. Peres ${ }^{3}$, P. \\ Alpuim ${ }^{*}, 1$ \\ 1 Departamento de Física, Universidade do Minho, Braga, Portugal \\ 2 Plasma Chemistry Research Center, CNR, Bari, Italy \\ ${ }^{3}$ Departamento de Física, Universidade de Aveiro, Aveiro, Portugal \\ ${ }^{4}$ Institute for Physics of Microstructures RAS, 603600 Nizhnij Novgorod GSP-105, Russia
}

PACS 71.23.-k, 73.20.Hb, 73.22.-f, 73.63.Bd, 78.55.-m, 78.67.Bf

Erbium-doped low-dimensional Si films with different microstructures were deposited by reactive magnetron sputtering on glass substrates by varying the hydrogen flow rate during deposition. Amorphous, micro- and nanocrystalline samples, consisting of $\mathrm{Si}$ nanocrystalls embedded in silicon-based matrices with different structures, were achieved with optical properties in the visible and IR depending on nanocrystalline fraction and matrix structure and chemical composition. Structural characterization was performed by X-ray diffraction in the grazing incidence geometry and Raman spectroscopy. The chemical composition was studied using RBS/ERD techniques. Spectroscopic ellipsometry was combined with the previous techniques to further resolve the film microstructure and composition. In particular, the distribution along the film thickness of the volume fractions of nanocrystalline/amorphous silicon and $\mathrm{SiO}_{\mathrm{x}}$ phases has been obtained.

In this contribution we discuss visible and infrared photoluminescence as a function of sample microstructure and of the oxygen/ hydrogen concentration ratio present in the matrix.

\section{Introduction}

Since the discovery of room temperature visible photoluminescence (PL) from porous silicon, research on nanostructured silicon-based materials has not ceased to increase with the aim of realizing efficient light emitters and amplifiers. Due to the interest in the ${ }^{4} \mathrm{I}_{13 / 2} \rightarrow{ }^{4} \mathrm{I}_{15 / 2}$ transition that occurs with emission of a photon at $\sim 1.54 \mu \mathrm{m}$, a wavelength that matches the minimum in the absorption spectrum of the silica-based optical communication fibers, a considerable amount of spectroscopic studies has been done in Er-doped $\mathrm{Si}$ [1-2]. When $\mathrm{Er}$ is incorporated in $\mathrm{c}-\mathrm{Si}_{\mathrm{B}} \mathrm{Er}^{3+}$ luminescence is quenched at temperatures well below room temperature, thus limiting the effectiveness of this system for optical-telecommunication devices Si [3-4]. Low-dimensional Si nanostruc- 
tures, where the Si nano-crystallites act as active species in the excitation process of rare earth ions, have been shown to be efficient $\mathrm{Er}^{3+}$ sensitizers with reduced PL thermal quenching. However, the fraction of $\mathrm{Er}^{3+}$ ions optically pumped is low when compared with the total amount of erbium present. It is known [1] that the exciton confined in the Si nanocrystal can have an important role in the erbium excitation mechanism, i.e., in Er-doped nanocrystalline silicon there is a correlation between the visible-PL emission from Si nanocrystals and the IR photoluminescence from $\mathrm{Er}^{3+}$ ions.

In our previous work it was observed that all the erbium-doped nanocrystalline silicon samples show the characteristic $1.54 \mu \mathrm{m}$ peak originating in the intra-atomic ${ }^{4} \mathrm{I}_{13 / 2} \rightarrow$ ${ }^{4} \mathrm{I}_{15 / 2}$ transition of the rare earth ion, independently of the erbium content. Furthermore, we found that samples with low doping level $(\approx 0.03$ at $\%)$ showed more intense $\mathrm{Er}^{3+}$ emission and also that the fraction of $\mathrm{Er}^{3+}$ ions not optically pumped forms $\mathrm{Er}_{2} \mathrm{Si}$ and/or $\mathrm{Er}_{2} \mathrm{O}_{3}$ compounds rather than metallic erbium [5].

In the present contribution we will study the visible and infrared PL of silicon thin films with a high amount of erbium and different oxygen/ hydrogen atomic ratio present in the matrix.

\section{Experimental}

Erbium doped micro- and nanocrystalline silicon (nc-Si) thin films were grown by reactive magnetron sputtering on glass substrates. The target was a c-Si wafer where some pieces of high purity (99.99\%) metallic erbium were added. All samples were grown in a hydrogen rich atmosphere. Oxygen was present at a contamination level during the deposition procedure. $R_{\mathrm{H} 2}$ is the hydrogen dilution of argon during deposition defined as $R_{\mathrm{H} 2}=F\left(\mathrm{H}_{2}\right) /\left[F\left(\mathrm{H}_{2}\right)+F(\mathrm{Ar})\right] \times 100 \%$ where the $F$ 's gas-flow rates. Erdoped silicon thin films that differ in the Er concentration, in the oxygen and hydrogen content and in the structure (crystallite fraction and nanocrystal size) were deposited. The different $\mathrm{O} / \mathrm{H}$ ratio has a strong impact on the film microstructure and consequently on the PL properties [6]. The chemical composition (see Table 1) was deter- 
mined by combining Rutherford backscattering spectroscopy (RBS) and elastic recoil detection (ERD) techniques. Structural characterization for evaluation of the size, $d$, and volume fraction, $X_{\mathrm{C}}$, of the crystallites (see Table 1) was carried out by microRaman spectroscopy under excitation with the $14.5 \mathrm{~nm}$ line of an $\mathrm{Ar}^{+}$laser.

Table 1 Properties of samples prepared by reactive magnetron sputtering.

\begin{tabular}{llllllllll}
\hline sample & $\begin{array}{l}T \\
\left({ }^{\circ} \mathrm{C}\right)\end{array}$ & $\begin{array}{l}\mathrm{rf} \\
(\mathrm{W})\end{array}$ & $\begin{array}{l}R_{12} \\
(\%)\end{array}$ & $\begin{array}{l}\mathrm{Er} \\
(\mathrm{at} \%)\end{array}$ & $\begin{array}{l}\mathrm{Si} \\
(\mathrm{at} \%)\end{array}$ & $\begin{array}{l}\mathrm{O} \\
(\mathrm{at} \%)\end{array}$ & $\begin{array}{l}\mathrm{H} \\
(\mathrm{at} \%)\end{array}$ & $\begin{array}{l}d \\
(\mathrm{~nm})\end{array}$ & $\begin{array}{l}X_{\mathrm{C}} \\
(\%)\end{array}$ \\
\hline Er34 & 50 & 80 & 63 & 0.03 & 72 & $<4$ & 27.5 & $<3 *$ & - \\
P7 & 300 & 40 & 63 & 2.8 & 75 & 20 & 2.2 & 7.2 & 34 \\
P13 & 300 & 80 & 63 & 0.55 & 70 & 9 & 17.7 & 0 & - \\
P16 & 300 & 80 & 75 & 0.60 & 65 & 10 & 24.9 & 7.5 & 62 \\
P19 & 200 & 40 & 63 & 1.41 & 74 & 14 & 9.4 & 7.4 & 43 \\
P28 & 150 & 40 & 63 & 1.81 & 71 & 15 & 11.5 & 7.0 & 59 \\
\hline
\end{tabular}

* Value determined by TEM analysis.

To analyze the Raman spectra computer simulations were used, treating the spectral profile as a superposition of the amorphous and crystalline parts. The crystalline profile was calculated on the basis of the strong phonon confinement model [7, 8] and a Gaussian profile was attributed to the amorphous transverse optical (TO) peak. Spectroscopic ellipsometry (SE) was also applied to analyze the film structure. SE spectra of the real, $\left\langle\varepsilon_{1}\right\rangle$, and imaginary, $\left\langle\varepsilon_{2}\right\rangle$, parts of the complex pseudo dielectric function, $\langle\varepsilon\rangle=\left\langle\varepsilon_{1}\right\rangle+\left\langle\varepsilon_{2}\right\rangle=(\mathrm{n}+\mathrm{ik})^{2}(\mathrm{n}$ is the refractive index and $\mathrm{k}$ is the extinction coefficient) were measured in the $0.75-5.5 \mathrm{eV}$ energy range using a phase modulated spectroscopic ellipsometer (UVISEL_Jobin Yvon) at an angle of incidence of $70.57^{\circ}$. The spectra were then analyzed using models based on the Bruggeman effective medium approximation (EMA) [9]. Since spectroscopic ellipsometry distinguishes the optical constants, namely the refractive index and extinction coefficient, of stoichiometric $\mathrm{SiO}_{2}$ from those of $\mathrm{SiO}$ and substoichiometric silicon oxides, this technique was used to determine the composition of the matrix where the crystals are embedded in. To describe the silicon matrix an EMA mixture of the dielectric function of nanocrystalline silicon (nc-Si) [10], microcrystalline silicon ( $\mu \mathrm{c}-\mathrm{Si}$ ) [11], amorphous silicon (a-Si) [12], $\mathrm{SiO}$ 
[13], and voids (modeling grain boundaries and microporosity) were used. For the erbium chemical environment, experimentally determined dielectric functions of silicide $\mathrm{ErSi}_{2}$ and oxide $\mathrm{Er}_{2} \mathrm{O}_{3}$ were used.

Steady state photoluminescence was generated by pumping with $325 \mathrm{~nm}$ and $514.5 \mathrm{~nm}$ light from $\mathrm{CW} \mathrm{He}-\mathrm{Cd}$ and $\mathrm{Ar}^{+}$lasers, respectively. The samples were mounted in the cold finger of a continuous-flow liquid He cryostat in vacuum and sample temperature was varied in the range from $\sim 7 \mathrm{~K}$ up to room temperature (RT). For the visible spectral range the PL was measured using a SPEX 1704 monochromator $\left(1 \mathrm{~m}, 1200 \mathrm{~mm}^{-1}\right)$ fitted with a cooled Hamamatsu R928 photomultiplier. For the infrared spectral region PL measurements were performed using a SPEX75 monochromator. The signal was detected by an Edinburgh Instruments germanium detector model EO-817, after excitation with the $514.5 \mathrm{~nm}$ line of an $\mathrm{Ar}^{+}$laser.

\section{Results and discussion}

Figure 1a shows the ellipsometric spectra of the imaginary part, $\left\langle\varepsilon_{2}\right\rangle$, of the pseudodielectric function for typical samples with a-Si matrix and with oxygen-rich Si matrix. The Er34 sample that has been extensively characterized in [10] has the peculiarity of containing nanocrystallites with a size $<3 \mathrm{~nm}$, as detected by transmission electron microscopy (TEM), embedded in an a-Si:H matrix with 27 at $\% \mathrm{H}$ content and without significant oxygen content $(<4$ at $\%)$. All other samples are richer in oxygen with $\mathrm{O}-$ content $>9$ at\%. Samples Er34 and P13 show the $3.6 \mathrm{eV}$-peak characteristic of amorphous material in their $\left\langle\varepsilon_{2}\right\rangle$ spectra obtained by SE, consistently with Raman data summarized in table 1 and spectra shown in in Figure 1b. However, contrary to the case of sample Er34, the ellipsometric analysis of sample P13 indicated that the amorphous matrix is oxygen rich (a-Si:H:O) with a Tauc band gap [14] of $1.96 \pm 0.01 \mathrm{eV}$.

Differently, the SE spectra of samples P7, P16, P19 and P28 show the $E_{1}$ and $E_{2}$ interband critical points $(C P \mathrm{~s})$ characteristic of crystalline silicon [15]. The ellipsometric analysis of these films, including the presence of the $C P$ s, indicate that they are microcrystalline with fine grains of size $<10 \mathrm{~nm}$, embedded in a oxygen rich matrix. Raman 
spectra of these samples show a very intense transverse optical (TO) mode around 520 $\mathrm{cm}^{-1}$ related to c-Si, and a Raman-crystallite size estimated to be approximately $7 \mathrm{~nm}$. The detailed ellipsometric analysis of the erbium bonding indicates that most of the erbium is incorporated into the silicon matrix as silicide $\mathrm{ErSi}_{2}$ or oxide $\mathrm{Er}_{2} \mathrm{O}_{3}$ depending on the composition and nanostructure of the Si matrix. The capability of ellipsometry for being sensitive to the erbium bonding is due to the different dielectric response of erbium silicide and erbium oxide [5].

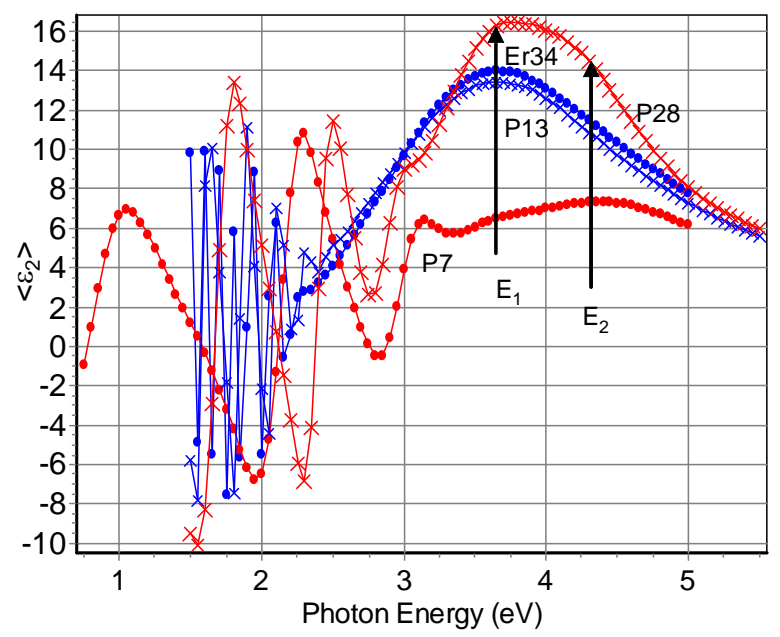

(a)

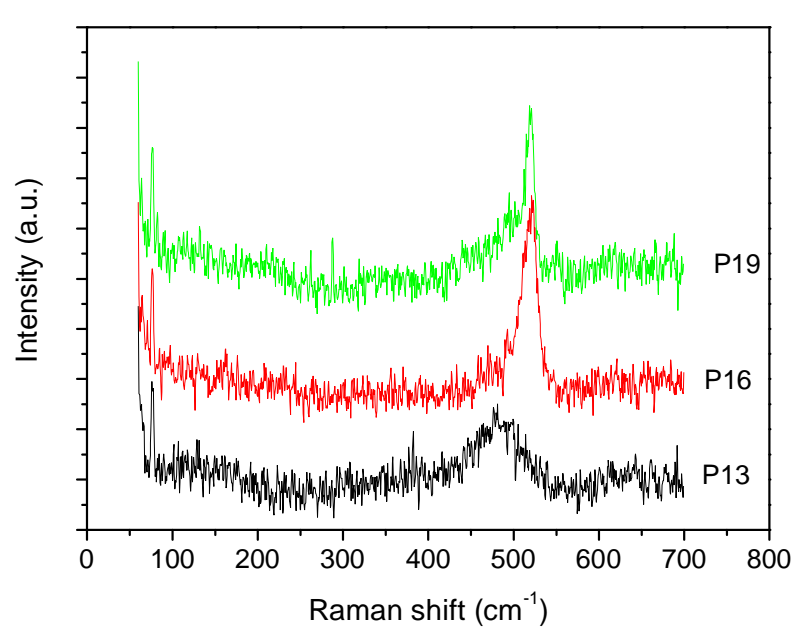

(b)

Fig 1 SE spectra of the imaginary part, $\left\langle\varepsilon_{2}\right\rangle$, of the pseudodielectric function (a) and Raman spectra (b) of selected amorphous and nanocrystalline Er-doped-thin silicon films

Figure $2 \mathrm{a}$ shows the temperature behavior of the $\mathrm{Er}^{3+}$ photoluminescence. Figure $2 \mathrm{~b}$ shows the room temperature PL spectra of the studied samples. All samples exhibit a characteristic band with a maximum around $1.535 \mu \mathrm{m}$, which is attributed to the $\mathrm{Er}^{3+}$ ions. As pointed out before the most intense $\mathrm{Er}^{3+} \mathrm{PL}$ was obtained in samples with a low fraction of small nanocrystals (Er34 and P13 samples). The temperature behavior was analyzed using the following fit function, which assumes a characteristic thermally activated excitation process, according to [16]: 


$$
I(T)=I(0)\left[1+C_{1} \exp \left(-E_{1} / k T\right)+C_{2} \exp \left(-E_{2} / k T\right)\right]^{-1}
$$

where $E_{1}$ is the activation energy for the high temperature range and $E_{2}$ is the corresponding energy for the low temperature region. The coefficients $C_{1}$ and $C_{2}$ take into account the excitation processes of Er ions. The low temperature erbium thermalization energy is around $5 \mathrm{meV}$ (between 4 and $6 \mathrm{meV}$ ) and the high temperature erbium thermalization energy is around $100 \mathrm{meV}$, as shown in table 2.

Although the less crystalline samples present a higher efficiency for erbium excitation than the more crystalline ones (P28 and P16), the PL quenching cannot be directly related with crystallinity, as can be seen in table II where the fitting parameters and the ratio of the PL intensity measured at RT and $10 \mathrm{~K}$ are presented. From tables 1 and 2 it can be concluded that the PL yield at RT decreases with the decrease of the hydrogen content. The beneficial role of hydrogen in improving optical and electrical quality of amorphous silicon films is well known, as it bonds to the silicon dangling bonds (DB's), passivating them. In the nanocrystalline films the defects, and in particular the DB`s, are located in the amorphous matrix, where the NC's are embedded, and at the grain boundaries (GB's). The role of atomic hydrogen in these films is to saturate both types of DB's and to passivate other defects. Therefore, the presence of non-radiative recombination pathways in samples with low hydrogen content is certainly responsible for the more important PL quenching observed there.

Table 2 Values of the curve-fitting parameters used to model temperature-dependent $\mathrm{Er}^{3+}-\mathrm{PL}$ behavior with eq. (1)

\begin{tabular}{cccccc}
\hline Sample & $C_{1}$ & $E_{1}(\mathrm{meV})$ & $C_{2}$ & $E_{2}(\mathrm{meV})$ & $R^{*}$ \\
\hline Er34 & 20 & 91 & 0.84 & 8 & 0.42 \\
$\mathrm{P} 16$ & 48.4 & 127 & 0.72 & 6 & 0.50 \\
$\mathrm{P} 13$ & 800 & 125 & 1.42 & 6 & 0.10 \\
$\mathrm{P} 28$ & 350 & 97 & 0.85 & 4 & 0.09 \\
\hline$* R=I_{P L} @ R T / I_{P L} @ 10 K$ & & &
\end{tabular}


Figure 3 shows the visible light-emission spectra obtained at $14 \mathrm{~K}$ for all the samples. Samples were optically pumped in the UV. It can be seen that the emission is mainly dominated by a broad band that spans from the blue into the infrared spectral regions. The band centered at $\sim 1.7 \mathrm{eV}$ is commonly attributed to the recombination of electronhole pairs confined in nc-Si [1]. All the emission spectra were maximized at this energy and taken under similar conditions. Interestingly two distinct spectral shapes can be observed. Namely, samples for which the dominant emission appears at energies lower than $\sim 2.5 \mathrm{eV}$ [Fig. 3 (a), (b)] and samples for which higher energy-emission bands are observed [Fig. 3 (c) - (f)].

In order to understand these differences we looked at the influence of the chemical matrix composition and crystalline fraction and crystallite size on the observed PL spectra. It is found that samples with the lowest oxygen content and smaller $(\sim 3.0 \mathrm{~nm})$ nanocrystallites (P13 and Er34) show intense PL peak centered at energies below $\mathrm{E}<2.5 \mathrm{eV}$. On the other hand, samples having high oxygen content and a crystalline volume fraction between $30-60 \%$, where the nanocrystals are embedded in an oxygen-rich silicon matrix, $\mathrm{SiO}_{\mathrm{x}}$, where $\mathrm{Er}_{2} \mathrm{O}_{3}$ is also present, show dominant PL emission at energies $\geq 2.5 \mathrm{eV}$. Samples P7, P16, P19 and P28 shown in Figures 3 (c), (d), (e) and (f) all belong to this group.

High energy visible-PL bands are frequently observed in samples with a larger crystalline volume fraction [17]. Besides the $\sim 1.7 \mathrm{eV}$ and $2.36 \mathrm{eV}$ emission bands related with excitons confined in $\mathrm{Si}$ nanocrystals, $\mathrm{c}-\mathrm{SiO}_{2}$ and $\mathrm{a}-\mathrm{SiO}_{2}$ are known to emit in the visible spectral region, namely at 2.6-2.7 eV and 2.1-2.2 eV [18-21]. Moreover the band around $2.2 \mathrm{eV}$ may arise from the oxygen-related luminescent centers located in the sub-oxide layer or in the amorphous $\mathrm{SiO}_{\mathrm{x}}$ network [22, 23]. In any case, high energy visible-PL bands (2.6-2.7 eV) are only present in oxygen-rich samples. 

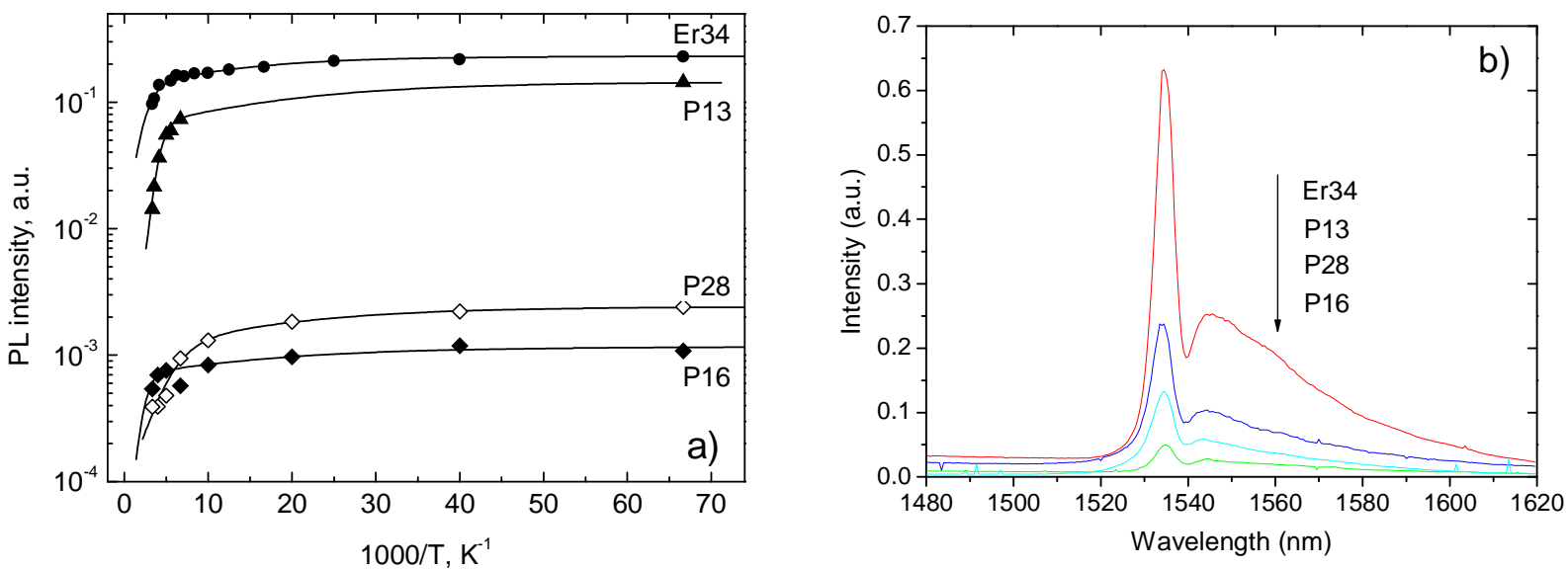

Fig 2 a) $\mathrm{Er}^{3+}$ photoluminescence intensity as a function of temperature for samples with a low fraction of very small nanocrystals (Er34 and P13) and with a higher fraction of larger nanocrystals (P16 and P28). b) PL spectra at $\sim 1.54 \mu \mathrm{m}$ measured at $20 \mathrm{~K}$ for the samples shown in a).

It has been observed that in Er doped samples a competition exists between silicon and erbium to get oxygen [24]. If hydrogen is also present in the matrix it will saturate the silicon DB and increase the PL yield (see Fig. 2).

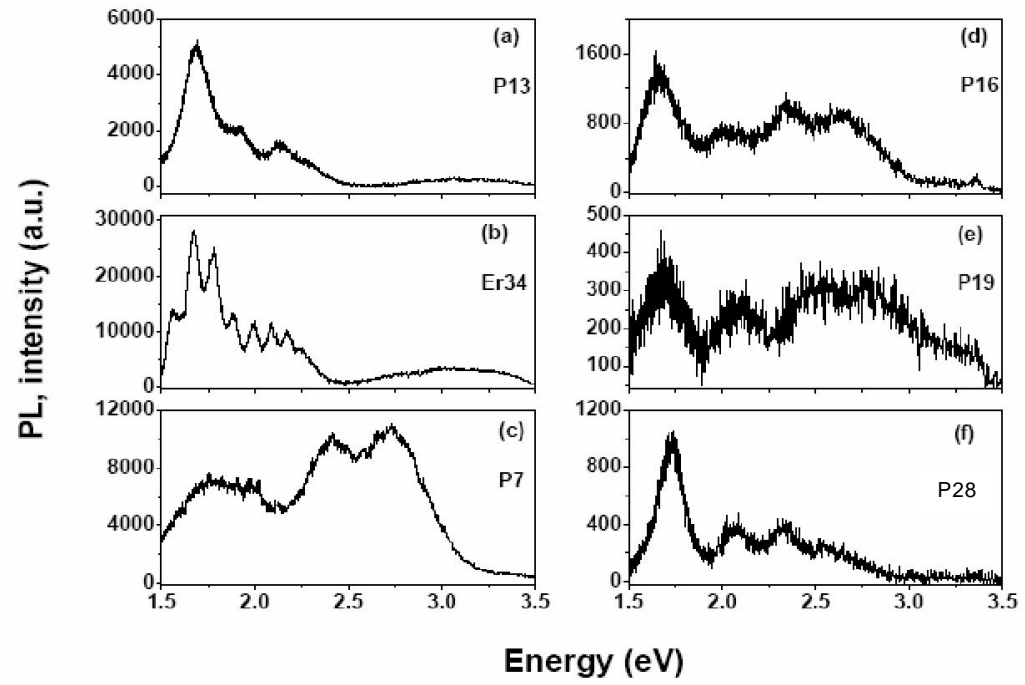

Fig 3 Visible photoluminescence spectra measured at $\sim 14 \mathrm{~K}$, using a He-Cd laser for optical pumping. 
These differences in matrix chemical composition can explain the high energy visible PL bands shown in Figure 3. In fact, the bands related with excitons confined in Si nanocrystals $(1.7 \mathrm{eV}$ and $2.36 \mathrm{eV})$ are present in the spectra of all high-crystallinefraction samples [Figure 3 (c) - (f)]. Simultaneously, oxygen-related luminescent centers are also clearly seen in those samples where the silicon nanocrystals are embedded in a $\mathrm{SiO}_{\mathrm{x}}$ matrix.

\section{Conclusions}

Erbium photoluminescence at $1.54 \mu \mathrm{m}$ without significant quenching up to temperatures $\sim 200-250 \mathrm{~K}$ was observed in RF-magnetron sputtered thin silicon films with different oxygen-to-hydrogen atomic concentration ratio, crystalline volume fraction and crystallite size. In these films, silicon nanocrystals are embedded in a Si-rich matrix whose nanostructure and chemical composition was shown to largely determine the photoluminescence efficiency. The $\mathrm{Er}^{3+}-\mathrm{PL}$ peak is more intense in samples with low erbium content $\left(\operatorname{Er}^{3+} \sim 0.03 \%\right)$ and small crystallite size $(<3 \mathrm{~nm})$; the PL quenching is lower in samples with high atomic-hydrogen content (samples P16 with 24.7at\% and Er34 with 27.5 at\%) due to a more complete hydrogen passivation of the deep defects that act as non-radiative recombination centers.

The observed visible luminescence after UV-optical pumping is dominated by the exciton-confined nc-Si recombination. Furthermore, broad emission bands in the orange and blue/UV are visible in samples where the matrix composition is oxygen-rich. From the visible PL-data it is possible to suggest that these emitting centers are due to oxygen-related defects. Ellipsometric studies performed on theses films identified an oxygen-rich structure of amorphous silicon as the main component of the matrix where nanocrystals are embedded.

\section{Acknowledgements}

One of the authors (M.P.) thanks FCT for a research grant. We acknowledge the support by FCT (POCTI/CTM/39395) and INTAS Project \#03-51-6486. 


\section{References}

[1] - M. Fujii, M. Yoshida, Y. Kanzawa, S. Hayashi, K. Yamamoto, Appl. Phys. Lett. 71, 1198 (1997).

[2] - P. G. Kik, M. L. Brongersma, A. Polman, Appl. Phys. Lett. 76, 2325 (2000).

[3] - S.-Y. Seo, J. H. Shin, Appl. Phys. Lett. 75, 4070 (1999)

[4] - P.G.Kik, A. Polman, J. Appl. Phys. 88, 1992 (2000)

[5] - M.F. Cerqueira, M. Stepikhova, M. Losurdo, A. Kozanecki, T. Monteiro, Optical Materials 28,836 (2006).

[6] - M.F. Cerqueira, M. Andritschky, L. Rebouta, J.A. Ferreira, M.F. Da Silva, Vacuum 46, 1385 (1995).

[7] - I.H. Campbell, P.M. Fauchet, Solid State Commun. 58, 739 (1986).

[8] - M. Yang, D. Huang, P. Hao, F. Zhang, X. Hou, X. Wang, J. Appl. Phys. 75, 651 (1994).

[9] - D. G. Bruggeman, Ann. Phys. (Leipzig) 24, 636 (1965).

[10] - M. Losurdo, M.M. Giangregorio, P. Capezzuto, G. Bruno, M.F. Cerqueira, E. Alves, M.V. Stepikhova, Appl. Phys. Lett. 82, 2993 (2003).

[11] - G.E. Jellison, M.F. Chisholm, S.M. Gorbatkin, Appl. Phys. Lett. 62, 3348 (1993.)

[12] - D.E. Aspnes, A.A. Studna, Phys. Rev. B 27, 985 (1983).

[13] - E.D. Palik, Handbook of Optical Constants of Solids II, Academic Press Inc., San Diego, CA, (1991).

[14] - J. Tauc, Optical Properties of solids, ed. F. Abeles (Amsterdam;North-Holland (1972)

[15] - P. Lautenschlager, M. Garriga, L. Vina, M. Cardona, Phys. Rev. B 36, 4821 (1987).

[16] - H. Przybylinska, W. Jantsch, Yu. Suprun-Belevitch, M. Stepikhova, L. Palmetshofer, G. Hendorfer, A. Kozanecki, R. J. Wilson and B. J. Sealy, Phys. Rev. B 54, 2532 (1996).

[17] - M.F. Cerqueira, T. Monteiro, M.V. Stepikhova, M. Losurdo, M.J. Soares, Isabel Gomes, Nanotechnology 15, 802 (2004).

[18] - J.H. Stahis, M.A. Kastner, Phys. Rev B 35, 2927 (1987).

[19] - R. Tohmon, H. Mizuno, Y. Ohki, K. Sasagane, K. Nagasawa, Y. Hama, Phys. Rev B 39, 1337 (1989).

[20] - R. Tohmon, Y. Shimogaichi, H. Mizuno, Y. Ohki, K. Nagasawa, Y. Hama, Phys. Rev. Let. 62, 1388 (1989). 
[21] - C. Itoh, T. Suzuki, N. Itoh, Phys. Rev B 41, 3794 (1990).

[22] - M. Wang, K. Chen, L. He, J. Xu, X. Huang, Appl. Phys. Lett. 73, 105 (1998).

[23] - A.V. Kabashin, M. Charbonneau-Lefort, M. Meunier, R. Leonelli, Appl. Surf. Sci. 168, 328 (2000).

[24] - M.F. Cerqueira, M. Losurdo, T. Monteiro, M. V. Stepikhova, J. Soares, M. Peres, E. Alves, O. Conde, Journal of Non-Crystalline Solids 352, 1148 (2006). 